\title{
EEG and autonomic responses during performance of matching and non-matching to sample working memory tasks with emotional content
}

\section{Ana Garcia*, Carlos Enrique Uribe, Maria Clotilde H. Tavares and Carlos Tomaz}

Laboratory of Neurosciences and Behavior, Department of Physiological Sciences, Institute of Biology, University of Brasília, Brasilia, Brazil

\author{
Edited by: \\ Antonella Gasbarri, University of \\ L'Aquila, Italy \\ Reviewed by: \\ Christa Mclntyre, University of Texas, \\ USA \\ Viviana Trezza, University Roma Tre \\ Italy \\ Stefano Puglisi-Allegra, Sapienza \\ Università di Roma, Italy \\ *Correspondence: \\ Ana Garcia, Laboratory of \\ Neuroscience and Behavior, \\ Department of Physiological \\ Sciences, Institute of Biology, \\ University of Brasília, Brasilia, Brazil. \\ e-mail: anacog@pobox.com
}

Working memory (WM) is a memory system responsible for the temporary storage of information and its utilization in problem solving. The central executive is theorized as the controller of storage functions that support WM. Neurophysiological data suggest that electroencephalographic (EEG) theta and alpha oscillations in frontal and midline regions are involved in neural communication between the central executive and storage functions during WM performance. Emotion is known to modulate several memory systems, including WM, through central and peripheral pathways. However, the physiological effect (EEG: autonomic nervous activity) of emotion over WM are not well described. In this study we aimed to identify physiological responses related to emotional WM performance. EEG (21 channels), heart rate (HR), and galvanic skin response (GSR) recordings were obtained from 54 volunteers while performing delayed matching and non-matching to sample tasks (DMTS/DNMTS). Emotional and neutral pictures from the International Affective Picture System and geometric figures were used as stimuli. As expected, WM performance was accompanied by presence of theta (frontal and midline electrodes) and alpha power (parietal electrodes). Beta and gamma oscillations were concentrated in frontopolar and left temporal regions. The DNMTS task was accompanied by higher increases in beta power, HR, and GSR compared to the DMTS task. Correlation analyses showed a positive tendency for gamma in the Fp2 site, ratio of LF/HF and skin conductance in both tasks. The $\mathrm{HR}$ results indicate an inverse reaction related to parasympathetic and sympathetic nervous system during the performance of the tasks. Taken together, our results contribute to elucidate the complex interactions between central and autonomic nervous systems in the modulation of emotional WM tasks.

Keywords: emotion, brain dynamics, heart rate, galvanic skin response

\section{INTRODUCTION}

Cognition and emotion are two, intrinsically connected, major elements of brain sciences, although often regarded as distinct. Many aspects of real life involve applying some kind of emotional criteria to make a decision or deal with a situation. Earlier studies have shown a high degree of connectivity between brain regions, such as frontal areas and limbic structures, as being central to cognitive-emotional interactions, critical for integration of information from different brain regions (e.g., Young et al., 1994). This relationship between cognition and emotion is performed via many different forms of interaction linked to the functional organization of the brain through many cognitive processes (Phelps, 2006; Pessoa, 2008) and also to body responses through the autonomic nervous system (Scherer, 1984; Lang, 1985; Umeno et al., 2002).

Working memory (WM) is a complex cognitive system designed to retain information until a certain problem has been solved (Baddeley, 1986). The proposal linked to this process is that the resolution of a problem involves the suitability of an overall decision, which requires the evaluation of experienced facts.
Moreover, WM alludes to the need to hold a stimulus in a priority state while information is manipulated to reach a certain goal, this being an essential part of the attention process. This focus of attention results in the explicit representation of the information in WM, and the simultaneous inhibition of irrelevant information (Hester and Garavan, 2005; Kim et al., 2005; Korsten et al., 2006; Pratt et al., 2011). The central executive is considered an attention system that controls the explicit manipulation of information during problem solving. It is comprised of the machinery necessary for manipulation of information in collaboration with specific components. One of its servers is the so-called episodic memory buffer, which binds information from multiple systems and recalls memories of personal experiences (Baddeley, 2000).

Electrophysiological measures, such those in electroencephalography (EEG), galvanic skin response (GSR), and heart rate (HR) variability, have been used to evaluate the mental workload and emotion recognition during dual tasks. Some of these studies assess decision-making and perception by means of association between the arousal of emotional content and increases in GSR (Laparra-Hernández et al., 2009). Others use emotional 
content to induce changes in autonomic nervous system, especially in HR and in GSR, in an attempt to define standards of autonomic responses for different types of emotion (Lee et al., 2005). Earlier studies indicate that cortical damage, especially to frontal lobes, may selectively attenuate GSR to psychologically relevant stimuli (Zahn et al., 1999). Additionally, cognitive functions such as attention and emotional memory encoding have been related to changes in parasympathetic activity (McCraty and Tomasino, 2006; Critchley, 2009). Some studies found a relationship between frontal and temporal cortice activity and cardiovascular responses associated with increasing emotional intensity, due to high arousal emotional content (Foster and Harrison, 2004).

The WM neural network has been the subject of behavioral, psychophysiological, and functional neuroimaging studies. Neuroimaging and EEG research has revealed the brain connections that support cognition, emotion, and the integration of information. The mainstays of this circuitry are the prefrontal cortex, including the central area, the occipito-parietal, and medial temporal regions (Curtis and D'Esposito, 2003; Osaka et al., 2003; Wager and Smith, 2003; Mizuhara et al., 2004; Erk et al., 2007; Gläscher et al., 2007; Pyka et al., 2009; Michels et al., 2010). Some studies were critical to sustain that the hippocampus modulates the WM system through temporal oscillations (Axmacher et al., 2008; van Vugt et al., 2010). As well as the functional association effects on WM performance, there is a general need to appraise the temporal organization of participant mechanisms using electrophysiological recordings, to demonstrate task-related brain activity (Jensen and Tesche, 2002; Schack and Sauseng, 2005; Kawasaki et al., 2010; Khader et al., 2010; Moran et al., 2010).

Behavioral procedures are important to elicit cognitive processes. The delayed matching to sample (DMTS) and delayed non-matching to sample (DNMTS) paradigms are widely used to assess WM (Tavares and Tomaz, 2002; Winocur and Hasher, 2004; Leiberg et al., 2006) and have been associated with emotional factors (Gasbarri et al., 2008; Satler and Tomaz, 2011). Both tasks involve memory and combining stimuli with a correct response. The DNMTS also requires increased attention to the subject chooses the novel stimulus (Elliot and Dolan, 1999). The difference in performance between stimuli categories, including emotional factors, represents the interaction between emotion and cognition. There is evidence for engagement of frontal and temporal areas of the brain, as well as premotor and occipital cortices, during DMTS and DNMTS performance (e.g., Elliot and Dolan, 1999; Lamar et al., 2004).

Several studies, designed for different purposes, have revealed relationships between cognition/emotion with EEG, HR, and GSR signals. For emotional WM, there are no studies including EEG brain mapping associated with autonomic responses. Therefore, the aim of the present study was to investigate electrophysiological responses during WM performance associated with emotional content processing. Delayed matching and non-matching to sample tasks were used with simultaneous EEG, GSR, and HR recording evaluations.

This approach will serve to elucidate: (1) central and peripheral correlates of WM performance in each of the tasks, (2) central and peripheral correlates of emotional content processing while performing a cognitive tests, and (3) central and peripheral correlates of the interactions between these two factors.

Several hypotheses will be tested: (1) Parasympathetic activity will be higher during DMTS task performance compared to DNMTS; (2) WM performance in both tasks will be facilitated by stimuli with emotionally arousing content; (3) Emotionally arousing content will engage sympathetic activity; and (4) Task and content effects over autonomic activity will interact in conditions of high cognitive demand and emotion arousal (e.g., DNMTS with emotional stimuli).

\section{MATERIALS AND METHODS PARTICIPANTS}

The subjects were 54 young adult ( 28 females) university students, with an age range from 18 to 28 and mean age of 21.31 years $(\mathrm{SD}=2.77)$. They were healthy, right-handed (assessed by the Edinburgh Inventory; Oldfield, 1971), with no history of neurological or psychiatric episodes. All subjects gave informed consent to a protocol approved by Ethics Committee of the Health Science Faculty, University of Brasilia, Brazil.

\section{DELAYED MATCHING AND NON-MATCHING TO SAMPLE TASK CONDITIONS}

This task had two phases in which each trial was intercalated by a delay $(1 \mathrm{~s})$. In the first phase, named acquisition, participants were presented with a sample stimulus (size $4 \mathrm{~cm} \times 4 \mathrm{~cm}$ ) in the center of a computer screen $\left(17^{\prime \prime}\right)$ for $2 \mathrm{~s}$. In the second phase, named testing, participants were randomly presented with two stimuli $(4 \mathrm{~cm} \times 4 \mathrm{~cm}$ each), one of which was the same as previously presented. In the second phase, the DMTS task required that participants choose the stimulus from the pair that matched the previously viewed target. In contrast, the DNMTS task required that participants choose the novel stimulus from the pair of stimuli after viewing the target (see Figure 1). An auditory feedback signal notified the participants if their response was correct (acute, for correct response, or bass, for incorrect or aborted response).

The emotional content of each stimulus was either emotional or non-emotional. Stimuli with emotional content was either Positive or Negative. Non-emotional stimuli was either Neutral or Geometric. Therefore, each stimulus belonged to one of four, mutually exclusive, categories (Positive, Negative, Neutral, or Geometric). The emotional and neutral stimuli were taken from the International Affective Picture System (IAPS; Lang et al., 1999), chosen according to the IAPS scale of valence and arousal. Other simple geometric figures (circles, triangles, etc.) were included to complete the set of stimuli. An equal number of pictures from each category were used. There were a total of 96 stimuli arranged in 48 different pairs, one set for DMTS and another for DNMTS, with the following arrangement: geometricgeometric; geometric-negative; geometric-neutral; geometricpositive; negative-geometric; negative-negative; negative-neutral; negative-positive; neutral-geometric; neutral-negative; neutralneutral; neutral-positive; positive-geometric; positive-negative; positive-neutral; and positive-positive. These tasks were performed using in house software, written for this purpose in Delphi language. 


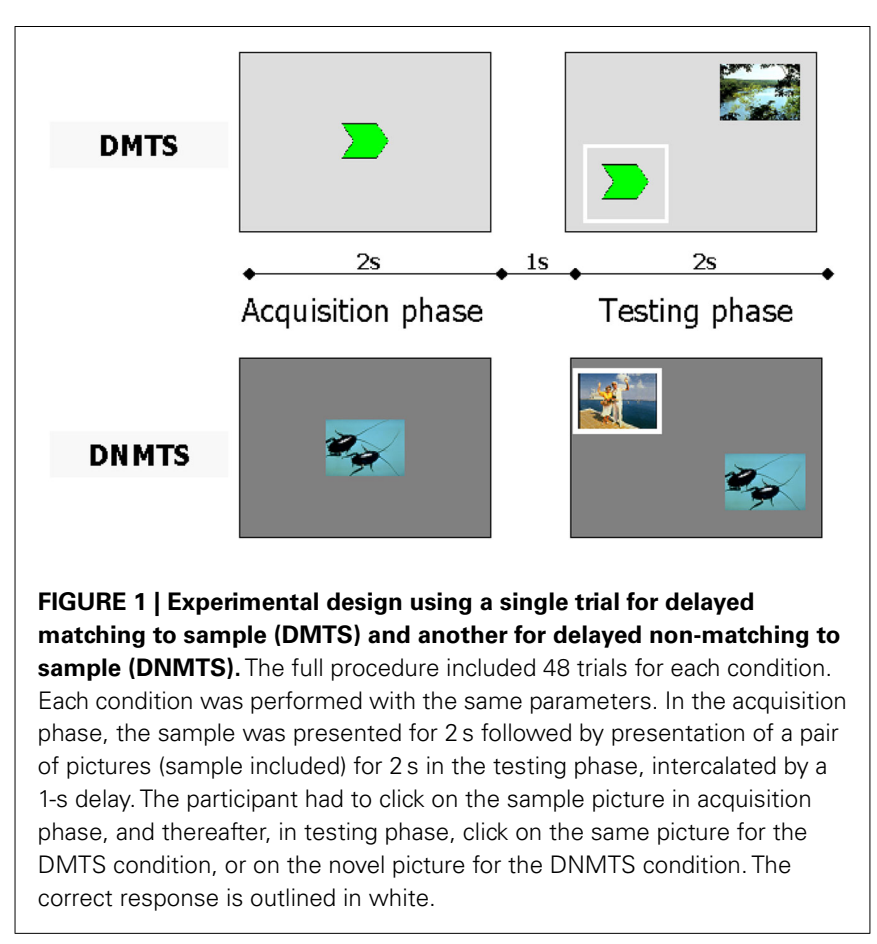

\section{DATA ACQUISITION AND PROCESSING}

Electroencephalographic data were collected from 21 channels placed on the scalp according to the 10-20 system, plus two reference electrodes on the right and left mastoid, fixed by a conductive paste (Ten20, Weaver and Company, USA). Each electrode site was previously prepared with an abrasive gel (Nuprep, Weaver and Company, USA) to improve conductance. HR was measured through two self-adhesive electrodes: one placed on the neck, over the jugular vein, and another placed on the middle of the left arm. GSR was recorded using two self-adhesive electrodes placed on the left hand, one on the palm and other on the back of the hand. The records were taken simultaneously at a sampling rate of $2000 \mathrm{~Hz}$ with an analog bandpass of 0.01$100 \mathrm{~Hz}$ using NeuronSpectrum-4/EP system (Neurosoft, Russia). Input impedances were maintained under $5 \mathrm{k} \Omega$ during the whole session.

All data were processed using customized Matlab scripts built to digitally separate into non-overlapping epochs time-locked to each task condition. EEG data were analyzed using the open source EEGLAB toolbox, version 9.0.4.5 (Delorme and Makeig, 2004; http://sccn.ucsd.edu/eeglab/). These epochs were submitted to an infomax algorithm to decompose into their independent components (ICA; Bell and Sejnowski, 1995). The components related to eye movement or blinking were removed from the original data, and the record was recalculated using the remaining components. HR and GSR data were filtered and processed for extraction of measures, also with customized Matlab scripts.

Heart rate processing was based on the detection of $\mathrm{R}$ peaks to calculate the indices of HR variability - rMSSD, the square root of the sum of the square of differences between adjacent RR intervals; SdNN, SD of RR periods; pNN50, percentage of the total number of all RR intervals of pairs of adjacent RR intervals differing more than $50 \mathrm{~ms}$ over the entire recording; LF/HF, ratio of low to high frequency power. The pNN50 helps assess parasympathetic activity: the smaller the percentage, the higher sympathetic activity. LF/HF reflects cardiac autonomic balance, greater values reflect sympathetic dominance, smaller values reflect parasympathetic dominance. GSR data were normalized to enable comparison of the values of each task condition for each subject. The indices calculated were SC, skin conductance mean of GSR values normalized; SCL, skin conductance level; and SCR, skin conductance resistance. These measures reflect sympathetic tone for general response (GSR) or were related to the stimulus presentation.

\section{PROCEDURE}

Participation entailed a single session of $2 \mathrm{~h}$ duration in the afternoon. By reading and signing the consent form, the participants were invited to make themselves comfortable in a chair in the recording room within a Faraday cage $(259 \mathrm{~cm} \times 223 \mathrm{~cm} \times 396 \mathrm{~cm})$, utilized for electromagnetic noise reduction. They were asked to avoid sudden movements or speaking during the task. The test was applied in both conditions, DMTS and DNMTS, in this order for each participant. Electrophysiological recordings were made simultaneously. The software registered correct responses, wrong responses, aborted trials (when participants did not give a response within $3 \mathrm{~s}$ ) and time taken for responses. The time of execution of the task depended on the time of response of each subject in each of the 48 trials. The room was devoid of luminance and noise during the task performance.

\section{STATISTICAL ANALYSIS}

Behavioral data were analyzed using repeated measures ANOVA (Condition $\times$ Category, $2 \times 4$ ). Statistical analysis on EEG data was performed with EEGLAB Toolbox. The paired $t$-test was used to compare the condition response for each HR index. A repeated measures ANOVA was also used for GSR data with a paired $t$-test per condition and stimulus. Degrees of freedom were corrected by Greenhouse-Geisser estimates of sphericity. However, the original degrees of freedom were reported for both ANOVA analyses. Pearson correlation coefficients were calculated among the electrophysiological parameters. Significance was defined as a $p$ value of less than 0.05 .

\section{RESULTS}

\section{BEHAVIORAL RESULTS}

Subjects showed an overall performance of $98.88 \%$ correct responses $(\mathrm{SD}=1.06)$ for the DMTS condition and $91.78 \%$ $(\mathrm{SD}=8.85)$ for the DNMTS condition (see Figure 2). Significant statistical differences were found for performance between conditions (DMTS $>$ DNMTS; $F_{1,53}=7.697 ; p=0.008$ ), between sample stimulus category $\left(F_{3,159}=25.632 ; p<0.001\right)$ and the interaction between condition and stimulus $\left(F_{3,159}=27.056 ; p<0.001\right.$; Table 1). Pair-wise comparisons showed statistical differences between Geometric vs. Neutral $(p<0.001)$, Geometric vs. Positive $(p=0.004)$, Geometric vs. Negative $(p<0.001)$, Neutral vs. Positive $(p<0.001)$ sample stimuli. Post hoc analyses showed that sample stimulus category effect was absent in DMTS condition $(p=0.095)$. On the order hand, within DNMTS condition the mean of scores of geometric category was significantly 


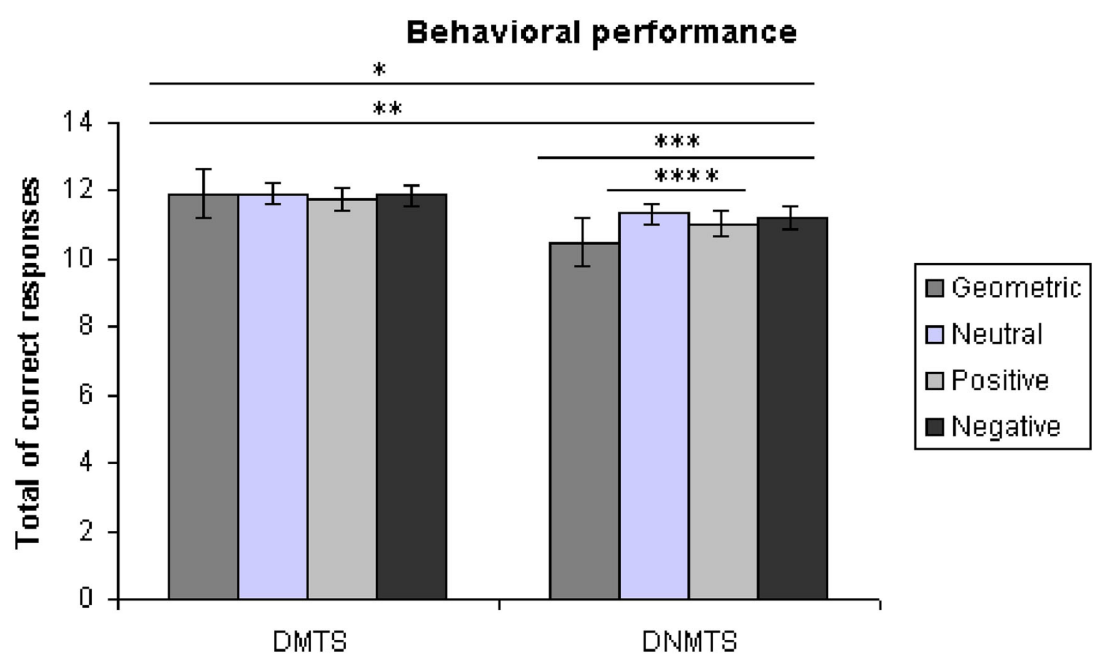

FIGURE 2 | Total of correct answers ${ }^{*}$ DMTS $>$ DNMTS, $p=0.008,{ }^{* *}$ geometric $<$ all other categories, $p<0.005,{ }^{* * *}$ geometric $<$ all other categories within DNMTS, $p<0.001,{ }^{* * * *}$ neutral $>$ positive within DNMTS, $p=0.001$.

Table 1 | Mean \pm SEM scores according to task condition and sample stimulus category.

\begin{tabular}{llll}
\hline Category & DMTS & DNMTS & Total \\
\hline Geometric & $11.907 \pm 0.048$ & $10.481 \pm 0.287^{\dagger}$ & $11.194 \pm 0.144^{* *}$ \\
Neutral & $11.926 \pm 0.045$ & $11.333 \pm 0.311^{\dagger \dagger}$ & $11.630 \pm 0.155$ \\
Positive & $11.759 \pm 0.074$ & $11.019 \pm 0.308$ & $11.389 \pm 0.154$ \\
Negative & $11.870 \pm 0.046$ & $11.222 \pm 0.315$ & $11.546 \pm 0.158$ \\
Total & $11.866 \pm 0.036^{*}$ & $11.014 \pm 0.301$ & \\
\hline
\end{tabular}

${ }^{*}$ DMTS $>$ DNMTS, $p=0.008,{ }^{*}$ Geometric $<$ all other categories, $p<0.005$, ${ }^{t}$ geometric < all other categories within DNMTS, $p<0.001,{ }^{t+}$ neutral $>$ positive within DNMTS, $p=0.001$.

lower than the other categories $(p<0.001)$, and the mean score for the neutral category was higher than the positive category $(p=0.001)$.

\section{EEG RESULTS}

Electroencephalographic data were filtered and divided into traditional frequency bands: Theta $(4-8 \mathrm{~Hz})$, Alpha $(8-13 \mathrm{~Hz})$, Beta $(13-30 \mathrm{~Hz})$, and Gamma $(30-70 \mathrm{~Hz})$. The frequency with the highest activity in each band was selected as the Reference Frequency. Table 2 shows the mean spectrum power of the frequency with the highest activity in each band. These values cannot be compared between bands, but provide a reference for each one. Figure 3 shows the topographic maps of activity for each condition.

\section{HR RESULTS}

Time domain and frequency domain measures showed significant differences between conditions. DMTS condition induced higher pNN50 ( $t=2.824 ; p=0.007)$, whereas DNMTS induced higher LFHF $(t=-2.673 ; p=0.010$; Table 3$)$. No differences were found for rMSSD or SdNN ( $t$ s $<1.681$; $p$ s $>0.99)$.
Table 2 | Mean spectrum power for each reference frequency.

\begin{tabular}{|c|c|c|c|c|c|}
\hline & Ref. Freq. & Location & DMTS & & DNMTS \\
\hline \multirow[t]{2}{*}{ Theta } & $5.859 \mathrm{~Hz}$ & $\mathrm{Fz}$ & 7.006 & $>$ & 6.841 \\
\hline & & $\mathrm{Cz}$ & 6.765 & $>$ & 6.701 \\
\hline \multirow[t]{5}{*}{ Alpha } & $9.766 \mathrm{~Hz}$ & $\mathrm{Fz}$ & 3.994 & $>$ & 3.875 \\
\hline & & $\mathrm{Cz}$ & 4.127 & $>$ & 4.071 \\
\hline & & $\mathrm{Pz}$ & 3.653 & $>$ & 3.534 \\
\hline & & Fp1 & 3.356 & $<$ & 3.901 \\
\hline & & $\mathrm{Fp} 2$ & 3.270 & $<$ & 3.829 \\
\hline \multirow[t]{3}{*}{ Beta } & $13.67 \mathrm{~Hz}$ & Fp1 & 1.908 & $<$ & 2.628 \\
\hline & & $\mathrm{Fp} 2$ & 1.730 & $<$ & 2.610 \\
\hline & & T3 & 0.845 & $>$ & 0.708 \\
\hline \multirow[t]{3}{*}{ Gamma } & $31.25 \mathrm{~Hz}$ & $\mathrm{Fp} 1$ & -5.034 & $<$ & -3.643 \\
\hline & & $\mathrm{Fp} 2$ & -4.803 & $<$ & -3.701 \\
\hline & & T3 & -4.429 & $>$ & -4.572 \\
\hline
\end{tabular}

Theta power was maximal in the midline for both conditions, with maximal expression in the $\mathrm{Fz}$ and $\mathrm{Cz}$ electrodes. DMTS condition was referenced slightly higher than DNMTS with dampening of this activity in medial regions (F3, F4, C3, C4, P3, P4), as shown in Figure 3. Alpha band activity extends toward the parietal central site $(P z)$ and also frontopolar $(F p 1, F p 2)$ regions, mainly in the DNMTS condition (Figure 3) maintaining the expression in the medial regions. Beta power was mostly concentrated in frontopolar regions. This band revealed statistically significant difference between conditions (DNMTS $>$ DMTS; $p<0.05$ ) in the Fp2 site. Beta activity was prominent at left temporal electrode (T3) for both groups (Figure 3). Gamma band resembled the topography found for Beta activity. Both bands were elevated in the DNMTS condition and highlight an important expression at the left temporal site (T3; Figure 3).

\section{GSR RESULTS}

Electrodermal measure did not present any differences, in general, between task condition DMTS: $0.018 \pm 0.137$, DNMTS: $0.0232 \pm 0.137$ (mean \pm SEM $)(t=-0.031 ; p=0.975)$. Considering the analysis per sample stimulus, significant statistical 


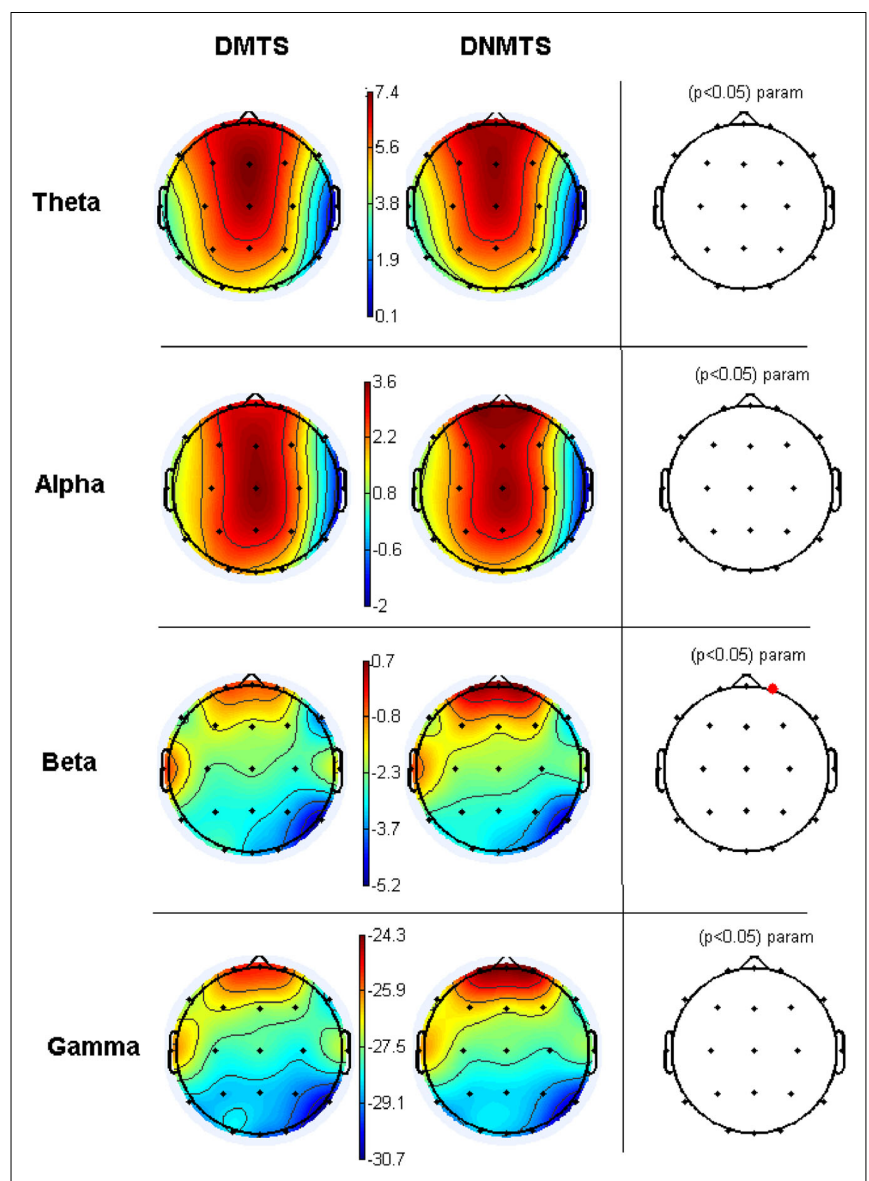

FIGURE 3 | Relative topographic power spectrum distribution for specified bands with each condition task, delayed matching to sample (DMTS) and delayed non-matching to sample (DNMTS), and statistical relevance related to tasks for each location. Theta activity - 4- to 8- $\mathrm{Hz}$; Alpha activity - 8- to $13-\mathrm{Hz}$; Beta activity - 13- to 30- $\mathrm{Hz}$; Gamma activity - 30- to $70-\mathrm{Hz}$. Red dot indicates significant difference $(p<0.05)$ in electrode location.

Table 3 | Mean \pm SEM of HR values according to task condition.

\begin{tabular}{llll}
\hline & DMTS & DNMTS & $\boldsymbol{p}$ \\
\hline rMSSD & $0.759 \pm 0.015$ & $0.751 \pm 0.014$ & 0.099 \\
SdNN & $0.048 \pm 0.004$ & $0.048 \pm 0.005$ & 0.996 \\
pNN50 & $0.080 \pm 0.012^{*}$ & $0.069 \pm 0.011$ & 0.007 \\
LFHF & $1.628 \pm 0.206$ & $1.940 \pm 0.214^{*}$ & 0.010
\end{tabular}

${ }^{*} p<0.05$.

differences were found for the SC index in the interaction between condition and sample stimulus $\left(F_{3,165}=4.027\right.$; $p=0.009)$. Post hoc analyses showed sample stimulus effect in negative category $(p=0.005)$. Statistical differences were marginal for SCL index between conditions (DMTS $<$ DNMTS; $F_{1,55}=3.754$; $p=0.058)$, and significant in the interaction between condition and sample stimulus $\left(F_{3,165}=6.626 ; p<0.001\right)$. Post hoc analyses showed a sample stimulus effect in the geometric $(p=0.004)$ and negative ( $p=0.004)$ categories. No significant statistical effect was found between stimuli for SCR index (see Table 4).
Table 4 | Mean \pm SEM of GSR values according to task condition and sample stimulus.

\begin{tabular}{llcl}
\hline & & DMTS & DNMTS \\
\hline SC & Geometric & $0.1161 \pm 0.0468$ & $0.0859 \pm 0.0405$ \\
& Neutral & $0.0981 \pm 0.0409$ & $0.1438 \pm 0.0390$ \\
& Positive & $-0.0101 \pm 0.1135$ & $0.1892 \pm 0.0564$ \\
& Negative* & $0.2344 \pm 0.0600$ & $0.0196 \pm 0.0553$ \\
SCL & Geometric & $0.3457 \pm 0.0148$ & $0.3649 \pm 0.0141$ \\
& Neutral & $0.3521 \pm 0.0152$ & $0.3512 \pm 0.0143$ \\
& Positive & $0.3534 \pm 0.0148$ & $0.3554 \pm 0.0139$ \\
SCR & Negative & $0.3413 \pm 0.0150$ & $0.3639 \pm 0.0155$ \\
& Geometric & $0.0004 \pm 0.0002$ & $0.0006 \pm 0.0003$ \\
& Neutral & $0.0003 \pm 0.0003$ & $0.0009 \pm 0.0003$ \\
& Positive & $0.0031 \pm 0.0021$ & $0.0009 \pm 0.0003$ \\
& Negative & $0.0026 \pm 0.0015$ & $0.0001 \pm 0.0003$
\end{tabular}

${ }^{*} p<0.05$.

\section{CORRELATION BETWEEN WM PERFORMANCE AND ELECTROPHYSIOLOGICAL RESPONSES}

Pearson bivariate correlations were calculated between all measures outlined above, taking into account an HR, a GSR, and an EEG parameter for all correlations and condition separately. A positive moderate correlation was found between $\mathrm{HR}$ (LF/HF) and Fp2 gamma power for DNMTS condition $(r=0.389 ; p=0.04)$, and between Fp2 gamma power and DNMTS condition score $(r=0.342 ; p=0.01)$. A weak correlation was seen for DMTS condition between SC and Fp2 gamma power $(r=0.126 ; p>0.01)$. Figure 4 illustrates the disposition of these correlations for each task condition. Considering sample stimulus category for performance and autonomic responses, correlations were calculated among scores and GSR's indices. A positive moderate correlation was found between scores and SCL of positive sample stimulus ( $r=0.345 ; p=0.011)$ for DMTS condition. No correlations were found between measures for DNMTS condition.

\section{DISCUSSION}

This study investigated EEG power concomitantly with autonomic reactions (GSR and HR) during WM performance associated with emotional content processing.

Behavioral results showed a high performance in both conditions, but statistically significant different scores between them (DMTS > DNMTS). The task comprises the ability to maintain the sample information online and recall this information after a delay according to a specific rule. Participants recalled emotional stimuli, negative and positive, with performance comparable to the neutral and geometric stimuli. The high correct performance found in both tasks limits us to emphasize a possible memory enhancing effect of emotional content, unlike that reported by earlier studies (Kensinger and Corkin, 2003), but consistent with others where negative emotional content were effective over the positive contents (Satler and Tomaz, 2011), mainly in DNMTS condition where more attention is required. However, we found a difference between tasks independent of the stimuli category. This can be explained by the fact that DNMTS requires the choice of the unfamiliar response, inhibiting the instinctively preferred familiar 
A

DMTS

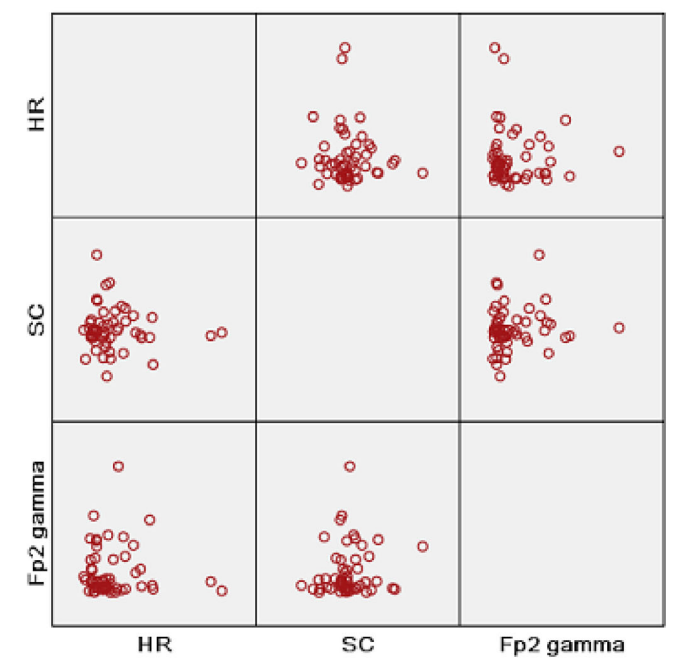

B

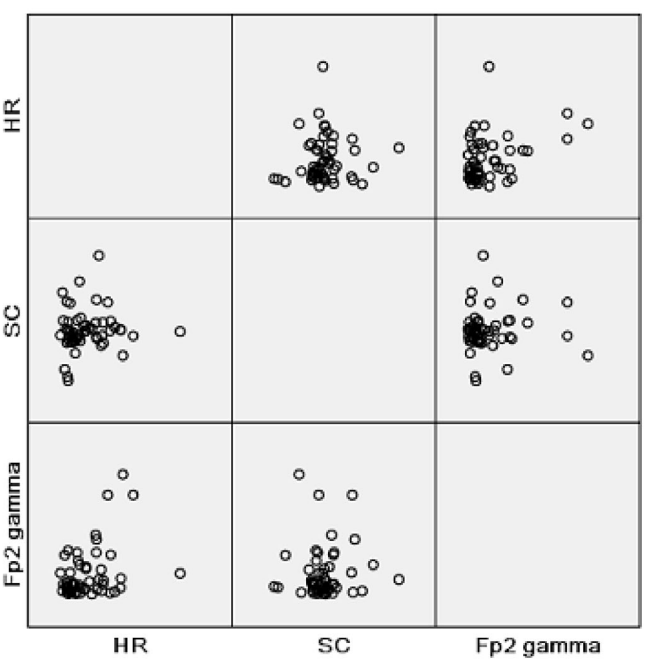

FIGURE 4 | Matrix scatter correlations, heart rate (HR) parameter with ratio of low to high frequency power (LF/HF), skin conductance mean index (SC) and gamma band power at right frontopolar location (Fp2 Gamma), in this order, in rows and columns for each condition task. (A) Condition DMTS; (B) condition DNMTS.

response. This aspect makes DNMTS performance lower than DMTS, supposedly due to attention demand (Elliot and Dolan, 1999; Lamar et al., 2004).

Electroencephalographic findings showed diversified rhythms which were present mainly in frontal and midline brain regions. Studies have shown that WM cannot be viewed as a single or dedicated system. A network of brain regions is essential for mediating goal-directed behavior, characteristic of this process (Collette and Van der Linden, 2002; D'Esposito, 2007). Theta activity was mainly observed in the prefrontal cortex and posterior association areas. These areas are strongly associated with central executive actions responsible for manipulation of information and planning (Baddeley, 2002; Jensen et al., 2007). Theta activity may have been induced by the task conditions performed. These results suggest that theta oscillations may play an important role in organizing the information network provided by WM, and this network operates as an integrated unit by means of synchronization in the theta band, consistent with earlier studies (Sarnthein et al., 1998; Raghavachari et al., 2001).

Alpha activity extended from frontal to posterior areas. Earlier findings suggest that alpha, associated with theta, oscillations reflect the transition of information from manipulation to maintenance state of WM tasks (Klimesch, 1999; Schack and Sauseng, 2005; Kawasaki et al., 2010; Moran et al., 2010). Likewise, DMTS tasks reproduces successful memory encoding and loading for processing WM. Increased alpha and theta power are regularly related to WM encoding, considering similar memory encoding processes to increasing WM load. This is consistent with the suggestion that alpha and theta oscillations modulate successful memory encoding (Khader et al., 2010).

The present study shows an increase of beta oscillations in frontal and temporal regions and includes task-related activity on the left hemisphere of the brain. This may be associated with attention demand, as representation of stimuli for DNMTS condition.
Frontal beta activation has been thought to be a memory representation of task stimuli (Dörfler et al., 2001; Vernon, 2005; Spitzer and Blankenburg, 2011). This band often appears with theta oscillations, WM load, and encoding processes (Babiloni et al., 2004).

Gamma band activity was distributed over frontal and temporal regions, and higher for DNMTS than DMTS condition. High frequency gamma oscillations have been considered an evidence of involvement in attention process (Mizuhara et al., 2004). Alternatively, gamma activity can reflect memory maintenance according to the short-term memory model, where each item of the memory set is encoded by cortical neurons at the gamma band frequency (Lisman and Idiart, 1995). Previous reports on gamma load-dependence in WM connected with theta oscillations also enhanced the idea that gamma oscillation increases as an expression of maintenance of the to-be-remembered stimuli (Howard et al., 2003; Jensen et al., 2007; Klimesch et al., 2007).

These findings also add some information on WM brain dynamics regarding temporal activity. In previous studies, beta rhythm has been observed at the left temporal and frontal electrodes during WM encoding phase (Onton et al., 2005; Pesonen et al., 2006; Altamura et al., 2010). In a general WM analysis, temporal activity was more prominent in the beta band than the gamma. These results attempt to others that indicate the gamma band leading WM load, with no reference to beta band in any manifestation (Axmacher et al., 2008; van Vugt et al., 2010).

Our results, therefore, show fluctuations in theta, alpha, beta, and gamma bands. Beta band was relevant in right frontopolar site as indicative of consistent task-related changes. Previous studies indicated oscillatory neuronal activity occurring across these frequency bands during WM load (Michels et al., 2010), and these fluctuations were also observed in tasks requiring organization and temporal segmentation of information (Howard et al., 2003). 
Some studies have indicated that emotions impact on the memory encoding process (Abe, 2001; Richter-Levin and Akirav, 2003), while underscoring the importance of amygdala-hippocampal connection in emotional memory load (Frank and Tomaz, 2003). Feasible hippocampal projection related to left temporal activity could be favored by subcortical structures responsible for emotion, such as amygdala, so as to better respond to emotional stimuli (McGaugh, 2000). Prior studies with WM tasks showed that bilateral prefrontal cortex activity reflected equally the emotional and WM task components (Gray et al., 2002). Earlier experiments showed a theta band manifestation for emotional content and gamma band for emotion arousal, both in frontal regions (Balconi and Pozzoli, 2009). These studies, along with our results, emphasize a significant electrophysiological interaction between cognition and emotion.

Autonomic responses showed an increased sympathetic activity (high LFHF and low pNN50) for the DNMTS condition. Results in HR index demonstrate a parasympathetic dominance in the DMTS condition, when a lower demand for cognitive functions is expected. Parasympathetic mediation of HRV has been associated with efficient attentional regulation and greater ability to inhibit inappropriate responses. Parasympathetic nervous system control of cardiovascular function, as well as activity of the prefrontal cortex, is associated with these inhibitory processes (Hansen et al., 2003). HR is a physiological variable controlled by sympathetic and parasympathetic activity. However GSR is not influenced by the parasympathetic nervous system, thereby making it a reliable index of sympathetic activity level (Venables, 1991), and is frequently used as an indirect measure of attention, or emotional arousal. The level of skin conductance was consistent with the cognitive effort considering the low performance for geometric sample stimuli and the arousal of negative emotional pictures. Equivalent emotional charge was applied in both conditions, thus no significant differences in GSR were found between conditions, but the expression

\section{REFERENCES}

Abe, K. (2001). Modulation of hippocampal long-term potentiation by the amygdala: a synaptic mechanism linking emotion and memory. J. Pharmacol. 86, 18-22.

Altamura, M., Goldberg, T. E., Elvevåg, B., Holroyd, T., Carver, F. W., Weinberger, D. R., and Coppola, R. (2010). Prefrontal cortex modulation during anticipation of working memory demands as revealed by magnetoencephalography. Int. J. Biomed. Imaging. doi: 10.1155/2010/840416.

Axmacher, N., Schmitz, D. P., Wagner, T., Elger, C. E., and Fell, J. (2008). Interactions between medial temporal lobe, prefrontal cortex, and inferior temporal regions during visual working memory: a combined intracranial EEG and functional magnetic resonance imaging study. J. Neurosci. 28, 7304-7312.

Babiloni, C., Babiloni, F., Carducci, F., Cincotti, F., Vecchio, F., Cola,
B., Rossi, S., Miniussi, C., and Rossini, P. M. (2004). Functional frontoparietal connectivity during short-term memory as revealed by high-resolution EEG coherence analysis. Behav. Neurosci. 118, 687-697.

Baddeley, A. (2000). The episodic buffer: a new component of working memory? Trends Cogn. Sci. (Regul. Ed.) 4, 417-423.

Baddeley, A. D. (1986). Working Memory. Oxford: Oxford University Press.

Baddeley, A. D. (2002). "Fractionating the central executive," in Principles of Frontal Lobe function, eds D. Stuss and R. T. Knight (New York: Oxford University Press), 246-260.

Balconi, M., and Pozzoli, U. (2009). Arousal effect on emotional face comprehension Frequency band changes in different time intervals. Physiol. Behav. 97, 455-446.

Bell, A. J., and Sejnowski, T. J. (1995). An information-maximization

of the sympathetic nervous system through a cardiovascular response was seen through the increase of GSR in the DNMTS condition.

The role of emotion was analyzed by evaluating human body response by means of HR and GSR measurements. Emotional content somehow modulates WM performance (Kensinger and Corkin, 2003; Levens and Phelps, 2008). Cognitive effort can also exert influence over emotion-related brain activation (Erk et al., 2007). Extrapolating, WM development has some benefit in the comprehension of emotions (Morra et al., 2011). This cross relation suggests that WM is susceptible to emotional content, in the same way that minimizing emotional reactions favors goal maintenance. Thus, the relationship between WM and emotion could be evaluated taking into account the arrangement of electrophysiological responses found while performing a WM task with emotional content. The correlations found between physiological variables were higher in the DNMTS condition, when attention demands are higher.

These results suggest an electrophysiological framework with interactions between brain dynamics and autonomic responses elicited by emotional engagement in a WM task. This adds elements to the relationship between cognitive processes and emotion. Future studies should evaluate the distinction over each emotion valence in task-relevant effects, assessing the specific contribution of positive, negative, and neutral, including abstract, representations.

\section{ACKNOWLEDGMENTS}

The present research was supported by a Conselho Nacional de Desenvolvimento Científico e Tecnológico (CNPq) scholarship to Carlos Uribe (140075/2008-9) and by a Fundação de Apoio à Pesquisa do Distrito Federal (FAPDF) grant to Carlos Tomaz (193.000.256/2007). We would like to thank Prof. Concepta McManus for her extensive english review and feedback on the manuscript.

approach to blind separation and blind deconvolution. Neural Comput. 7, 1129-1159.

Collette, F., and Van der Linden, M. (2002). Brain imaging of the central executive component of working memory. Neurosci. Biobehav. Rev. 26, 105-125.

Critchley, H. D. (2009). Psychophysiology of neural, cognitive and affective integration: fMRI and autonomic indicants. Int. J. Psychophysiol. 73, 88-94.

Curtis, C. E., and D’Esposito, M. (2003). Persistent activity in the prefrontal cortex during working memory. Trends Cogn. Sci. (Regul. Ed.) 7, 415-423.

Delorme, A., and Makeig, S. (2004). EEGLAB: an open source toolbox for analysis of single-trial EEG dynamics. J. Neurosci. Methods 134, 9-21.

D’Esposito, M. (2007). From cognitive to neural models of working memory. Philos. Trans.
R. Soc. Lond. B Biol. Sci. 362, 761-772.

Dörfler, T., Simmel, A., Schleif, F.M., and Sommerfeld, E. (2001). "Complexity-dependent synchronization of brain subsystems during memorization", in Proceedings of the 17th Meeting of the International Society for Psychophysics (Leipzig: Pabst Publishing), 343-348.

Elliot, R., and Dolan, R. J. (1999). Differential neural responses during performance of matching and nonmatching to sample tasks at two delay intervals. J. Neurosci. 19, 5066-5073.

Erk, S., Kleczar, A., and Walter, H. (2007). Valence-specific regulation effects in a working memory task with emotional context. Neuroimage 37, 623-632.

Foster, P. S., and Harrison, D. W. (2004). The covariation of cortical electrical activity and cardiovascular responding. Int. J. Psychophysiol. 52, 239-255. 
Frank, J. E., and Tomaz, C. (2003). Lateralized impairment of the emotional enhancement of verbal memory in patients with amygdalahippocampus lesion. Brain Cogn. 52, 223-230.

Gasbarri, A., Pompili, A., d'Onofrio, A., Cifariello, A., Tavares, M. C., and Tomaz, C. (2008). Working memory for emotional facial expressions: role of the estrogen in young women. Psychoneuroendocrinology 33, 964-972.

Gläscher, J., Rose, M., and Büchel, C. (2007). Independent effects of emotion and working memory load on visual activation in the lateral occipital complex. J. Neurosci. 27, 4366-4373.

Gray, J. R., Braver, T. S., and Raichle, M. E. (2002). Integration of emotion and cognition in the lateral prefrontal cortex. Proc. Natl. Acad. Sci. U.S.A. 99, 4115-4120.

Hansen, A. L., Johnsen, B. H., and Thayer, J. F. (2003). Vagal influence on working memory and attention. Int. J. Psychophysiol. 48, 263-274.

Hester, R., and Garavan, H. (2005). Working memory and executive function: the influence of content and load on the control of attention. Mem. Cognit. 33, 221-233.

Howard, M. W., Rizzuto, D. S., Caplan, J. B., Madsen, J. R., Lisman, J., Aschenbrenner-Scheibe, R., SchulzeBonhage, A., and Kahana, M. J. (2003). Gamma oscillations correlate with working memory load in humans. Cereb. Cortex 13, 1369-1374.

Jensen, O., Kaiser, J., and Lachaux, J. (2007). Human gamma-frequency oscillations associated with attention and memory. Trends Neurosci. 30, 317-324.

Jensen, O., and Tesche, C. D. (2002). Frontal theta activity in humans increases with memory load in a working memory task. Eur. J. Neurosci. 15, 1395-1399.

Kawasaki, M., Kitajo, K., and Yamaguchi, Y. (2010). Dynamic links between theta executive functions and alpha storage buffers in auditory and visual working memory. Eur. J. Neurosci. 31, 1683-1689.

Kensinger, E. A., and Corkin, S. (2003). Effect of negative emotional content on working memory and long-term memory. Emotion 3, 378-393.

Khader, P. H., Jost, K., Ranganath, C., and Rösler, F. (2010). Theta and alpha oscillations during workingmemory maintenance predict successful long-term memory encoding. Neurosci. Lett. 468, 339-343.

Kim, S., Kim, M., and Chun, M. M. (2005). Concurrent working memory load can reduce distraction.
Proc. Natl. Acad. Sci. U.S.A. 102, 16524-16529.

Klimesch, W. (1999). EEG alpha and theta oscillations reflect cognitive and memory performance: a review and analysis. Brain Res. Rev. 29, 169-195.

Klimesch, W., Sauseng, P., and Hanslmayr, S. (2007). EEG alpha oscillations: the inhibition-timing hypothesis. Brain Res. Rev. 53, 63-88.

Korsten, N. J. H., Fragopanagos, N., Hartley, M., Taylor, N., and Taylor, J. G. (2006). Attention as a controller. Neural Netw. 19, 1408-1421.

Lamar, M., Yousem, D. M., and Resnick, S. M. (2004). Age differences in orbitofrontal activation: an fMRI investigation of delayed match and non-match to sample. Neuroimage 21, 1368-1376.

Lang, P. J. (1985). "Cognition in emotion: concept and action," in Emotions, Cognition, and Behavior, eds E. Carroll, E. Izard, E. Jerome Kagan, and R. Zajonc (New York, NY: Cambridge University Press), 192-226.

Lang, P. J., Bradley, M. M., and Cuthbert, B. N. (1999). International Affective Picture System (IAPS): Technical Manual and Affective Ratings. Center for Research in Psychophysiology, University of Florida, Gainesville.

Laparra-Hernández, J., Belda-Lois, J. M., Medina, E., Campos, N., and Poveda, R. (2009). EMG and GSR signals for evaluating user's perception of different types of ceramic flooring. Int. J. Ind. Ergon. 39, 326-332.

Lee, C. K., Yoo, S. K., Park, Y. J., Kim, N. H., Jeong, K. S., and Lee, B. C. (2005). "Using neural network to recognize human emotions from heart rate variability and skin resistance," in Proceedings of the 2005 IEEE Engineering in Medicine and Biology 27th Annual Conference, Shanghai.

Leiberg, S., Kaiser, J., and Lutzenberger, W. (2006). Gamma-band activity dissociates between matching and nonmatching stimulus pairs in an auditory delayed matchingto-sample task. Neuroimage 30, 1357-1364.

Levens, S. M., and Phelps, E. A. (2008). Emotion processing effects on interference resolution in working memory. J. Emot. 8, 267-280.

Lisman, J. E., and Idiart, M. A. (1995). Storage of $7 \pm 2$ short-term memories in oscillatory subcycles. Science 267, 1512-1515.

McCraty, R. M., and Tomasino, D. (2006). "Emotional stress, positive emotions, and psychophysiological coherence," in Stress in Health and
Disease, eds B. B. Arnetz and R. Ekman (Weinheim: Wiley-VCH), 360-383.

McGaugh, J. L. (2000). Memory - a century of consolidation. Science 287, 248-251.

Michels, L., Bucher, K., Lüchinger, R. Klaver, P., Martin, E., Jeanmonod, D., and Brandeis, D. (2010). Simultaneous EEG-fMRI during a working memory task: modulations in low and high frequency bands. PLoS ONE 5, e10298. doi:10.1371/journal.pone.0010298

Mizuhara, H., Wang, L-Q., Kobayashi, K., and Yamaguchi, Y. (2004). Emergence of a long-range coherent network associated with frontal midline theta during a mental task in human: a study of simultaneous EEG and fMRI. Neuroreport 15, 1233-1238.

Moran, R. J., Campo, P., Maestu, F. Reilly, R. B., Dolan, R. J., and Strange, B. A. (2010). Peak frequency in the theta and alpha bands correlates with human working memory capacity. Front. Hum. Neurosci. 4:200. doi:10.3389/fnhum.2010.00200

Morra, S., Parrella, I., and Camba, R. (2011). The role of working memory in the development of emotion comprehension. Br. J. Dev. Psychol. 29, 744-764.

Oldfield, R. C. (1971). The assessment and analysis of handedness: the Edinburgh inventory. Neuropsychologia 9, 97-113.

Onton, J., Delorme, A., and Makeig, S. (2005). Frontal midline EEG dynamics during working memory. Neuroimage 27, 341-356.

Osaka, M., Osaka, N., Kondo, H., Morishita, M., Fukuyama, H., Aso, T. and Shibasaki, H. (2003). The neural basis of individual differences in working memory capacity: an fMRI study. Neuroimage 18, 789-797.

Pesonen, M., Bjornberg, C. H. Hamalainen, H., and Krause, C. M. (2006). Brain oscillatory $1-30 \mathrm{~Hz}$ EEG ERD/ERS responses during the different stages of an auditory memory search task. Neurosci. Lett. 399, 45-50.

Pessoa, L. (2008). Opinion: on the relationship between emotion and cognition. Nat. Rev. Neurosci. 9, 148-158.

Phelps, E. A. (2006). Emotion and cognition: Insights from studies of the human amygdala. Annu. Rev. Psychol. 57, 27-53.

Pratt, N., Willoughby, A., and Swick, D. (2011). Effects of working memory load on visual selective attention: behavioral and electrophysiological evidence. Front. Hum. Neurosci. 5:57. doi:10.3389/fnhum.2011.00057
Pyka, M., Beckmann, C. F., Schöning, S., Hauke, S., Heider, D., Kugel, H., Arolt, V., and Konrad, C. (2009). Impact of working memory load on fMRI resting state pattern in subsequent resting phases. PLoS ONE 4, e7198. doi:10.1371/journal.pone.0007198

Raghavachari, S., Kahana, M. J., Rizzuto, D. S., Caplan, J. B., Kirschen, M. P., Bourgeois, B., Madsenm, J. R., and Lisman, J. E. (2001). Gating of human theta oscillations by a working memory task. J. Neurosci. 21, 3175-3183.

Richter-Levin, G., and Akirav, I. (2003). Emotional tagging of memory formation - in the search for neural mechanisms. Brain Res. Rev. 43, 247-256.

Sarnthein, J., Petsche, H., Rappelsberger, P., Shaw, G. L., and von Stein, A. (1998). Synchronization between prefrontal and posterior association cortex during human working memory. Proc. Natl. Acad. Sci. U.S.A. 95, 7092-7096

Satler, C., and Tomaz, C. (2011). Emotional working memory in Alzheimer's disease patients. Dement. Geriatr. Cogn. Disord. Extra 1, 124-138.

Schack, B., and Sauseng, P. (2005). Phase synchronization between theta and upper alpha oscillations in a working memory task. Int. J. Psychophysiol. 57, 105-114.

Scherer, K. R. (1984). "On the nature and function of emotion: a component process approach," in Approaches to Emotion, eds K. P. Scherer and P. Ekman (Hillsdale, NJ: Lawrence Erlbaum Associates), 293-317.

Spitzer, B., and Blankenburg, F. (2011). Stimulus-dependent EEG activity reflects internal updating of tactile working memory in humans. Proc. Natl. Acad. Sci. U.S.A. 108, 8444-8449.

Tavares, M. C., and Tomaz, C. (2002). Working memory in capuchin monkeys (Cebus apella). Behav. Brain Res. 131, 131-137.

Umeno, K., Hori, E., Tabuchi, E., Takakura, H., Miyamoto, K., Ono, T., and Nishijo, H. (2002). Gamma-band EEGs predict autonomic responses during mental arithmetic. Neuroreport 14, 477-480.

van Vugt, M. K., Schulze-Bonhage, A., Litt, B., Brandt, A., and Kahana, M. J. (2010). Hippocampal gamma oscillations increase with memory load. J. Neurosci. 30, 2694-2699.

Venables, P. H. (1991). Autonomic activity. Ann. N. Y. Acad. Sci. 620, 191-207. 
Vernon, D. J. (2005). Can neurofeedback training enhance performance? An evaluation of the evidence with implications for future research. Appl. Psychophysiol. Biofeedback 30, 347-364.

Wager, T. D., and Smith, E. E. (2003). Neuroimaging studies of working memory: a meta-analysis. Cogn. Affect. Behav. Neurosci. 3, 255-274.

Winocur, G., and Hasher, L. (2004). Age and time-of-day effects on learning and memory in a non-matchingto-sample test. Neurobiol. Aging 25, 1107-1115.

Young, M. P., Scannell, J. W., Burns, G. A., and Blakemore, C. (1994). Analysis of connectivity: neural systems in the cerebral cortex. Rev. Neurosci. 5, 227-249.

Zahn, T. P., Grafman, J., and Tranel, D. (1999). Frontal lobe lesions and electrodermal activity: effects of significance. Neuropsychologia 37, 1227-1241.
Conflict of Interest Statement: The authors declare that the research was conducted in the absence of any commercial or financial relationships that could be construed as a potential conflict of interest.

Received: 07 October 2011; accepted: 01 December 2011; published online: 21 December 2011.

Citation: Garcia A, Uribe CE, Tavares MCH and Tomaz C (2011) EEG and autonomic responses during performance of matching and non-matching to sample working memory tasks with emotional content. Front. Behav. Neurosci. 5:82. doi: 10.3389/fnbeh.2011.00082

Copyright (c) 2011 Garcia, Uribe, Tavares and Tomaz. This is an open-access article distributed under the terms of the Creative Commons Attribution Non Commercial License, which permits non-commercial use, distribution, and reproduction in other forums, provided the original authors and source are credited. 